\title{
An Internet Learning Environment for Collaborative Notetaking
}

\author{
Weijen Hsueh and Chih-Wei Hue \\ Department of Psychology, National Taiwan University
}

\begin{abstract}
An Internet learning environment was created to allow users to write studying notes in collaboration. Effective study procedure was incorporated into the system to promote collaboration among users. The procedure is as the follows: the members (1) preview learning materials and ask questions about the important information the materials contained, (2) discuss and divide the materials into a few shares, (3) read the assigned materials to answer the questions raised, and write the answers on notes, (4) review and discuss the notes took by each member, and integrate the notes into an unified copy. A questionnaire study was conducted to collected users' attitudes toward the system.
\end{abstract}

\section{1: Introduction}

College students in Taiwan usually study in groups, and the members of a studying group will not only share their notes took in class, but also divide the learning materials into equal shares, and each member needs to study only a share. After studying individually, they will exchange their studying notes so that each member will possess a copy of the studying notes created together by all the members of the group. In the following text, this type of notes will be referred to as type-D studying notes. Obviously, "D" stands for job division.

There are shortcomings associated with type-D notetaking behavior. For example, the quality of the notes is different from one person to another because of the individual difference in notetaking ability. Moreover, dividing learning materials into a number of shares is likely to detach the information contained in a share from its context, and this will hurt a learner's understand of the information.

The present research designed an Internet environment allowing users to study the materials contained and to take notes in groups. The users of the system follow a 4stage notetaking process modified from the procedure of a common effective study program (e.g., PQRST and PR4R) to create notes. The followings are the 4 notetaking stages.

1. Preview and Questioning: The members of a studying group preview the materials under study, and raise questions concerning the important messages the materials intended to convey.

2. Assignment: The members of a group discuss how to divide the materials of a learning unit into equal shares, and each member adopts a share as his/her responsibility of study.

3. Read: A member reads the materials assigned to him/her. In particular, s/he should read to answer the questions raised by all the members in the Preview and Questioning stage, and note down the answers.

4. Discussion and Integration: All the members review and discuss the notes took by each member, and a member should reread the learning materials in consideration of the other members' comments, and revise his/her notes. Finally, after discussion, the notes are integrated into a unified copy, and each 
member can get a copy of it.

The users work together discussing and exchanging ideas, throughout entire notetaking process, we argued the system creates a type-C notetaking environment, where "C" stands for collaboration. Moreover, although each member of a group read only a part of the materials, s/he reads to answer the questions raised by all the members the studying group. In the Integration stage, if there is misunderstanding of the materials or missing important information in an user's notes, the other users are likely to spot it out because their knowledge of learning materials is complement to each other. The system is built on Internet and the users are not limited to use the information stored in the system to answer questions, thus there is possibility that the users can develop knowledge beyond their teacher's assignment. Thus, not only a user of the system enjoys the advantages, but $\mathrm{s} /$ he also avoids the disadvantages of the type-D notetaking process.

\section{2: The Learning Environment}

The system mentioned above have been implemented in a one-year experimental psychology course which consists of 23 learning units. Teaching materials of the course consist of texts, figures, videos, computer programs designed to run psychological experiments, however, only the texts and figures were included in the system.

Users can access the materials through the system in the form of studying group. Similar to other systems with notetaking functions (e.g., CoVis), a user can put highlights and "stickers" on the materials (Fig. 1). Each user highlights with a color of his/her choice. A user can write comments and summaries of the learning material, or s/he can post figures and IP links on a sticker.

To make sure that all the members of a group participate in discussion, a table is designed to keep track which notetaking stage the group is in and in a particular stage which member has asked question or given comment and which has not. When all the members have participated in discussion, the system can be switched to the next notetaking stage upon the learners' request.

\section{3: Students' Attitudes Toward the Learning Environment}

A preliminary study of 50 subjects' attitudes toward the learning environment has been collected. The subjects were divided into small groups (4 to 6 a group), and in a 1-hour research session, they received a 15-minute illustration as to how the system operated, and followed by a 30 -minute practice, and in the last 15 minutes, the subjects were asked to fill a questionnaire.

The research results indicated that the subjects' had positive attitudes toward the system. Eighty-four percents of them agreed that the system could promote ideas exchange among group members, $82 \%$ believed that the system could enhance a learner's understanding of the learning materials. More than $80 \%$ of the subjects indicated that they are willing to use the system to take collective notes if they are going to take notes. And $82 \%$ of the subjects indicated that they are willing to introduce the system to their friends as a means to study.

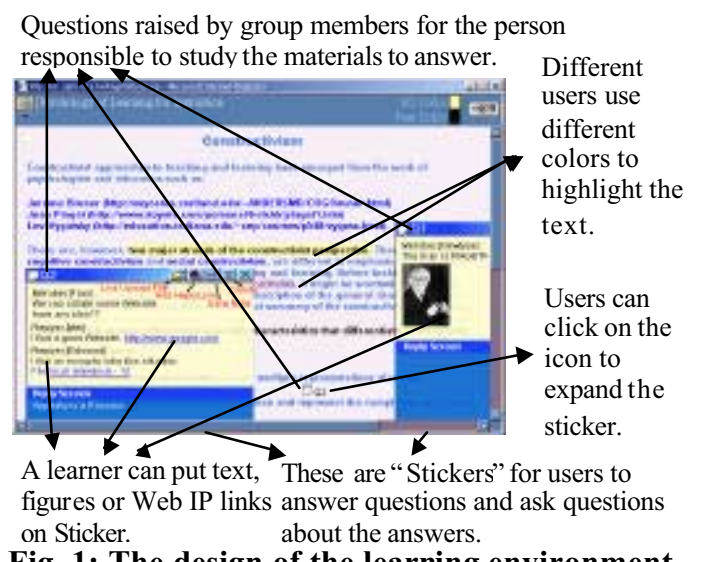

Fig. 1: The design of the learning environment. 